\title{
Non-linear unsteady inverse boundary problem for heat conduction equation
}

\author{
MAGDA JOACHIMIAK* \\ MICHAŁ CIAŁKOWSKI
}

Poznań University of Technology, Chair of Thermal Engineering, Piotrowo 3, 60-965 Poznań

\begin{abstract}
Direct and inverse problems for unsteady heat conduction equation for a cylinder were solved in this paper. Changes of heat conduction coefficient and specific heat depending on the temperature were taken into consideration. To solve the non-linear problem, the Kirchhoff's substitution was applied. Solution was written as a linear combination of Chebyshev polynomials. Sensitivity of the solution to the inverse problem with respect to the error in temperature measurement and thermocouple installation error was analysed. Temperature distribution on the boundary of the cylinder, being the numerical example presented in the paper, is similar to that obtained during heating in the nitrification process.
\end{abstract}

Keywords: Inverse problem; Sensitivity of the solution to the inverse problem; Application of Chebyshev polynomials

\section{Nomenclature}

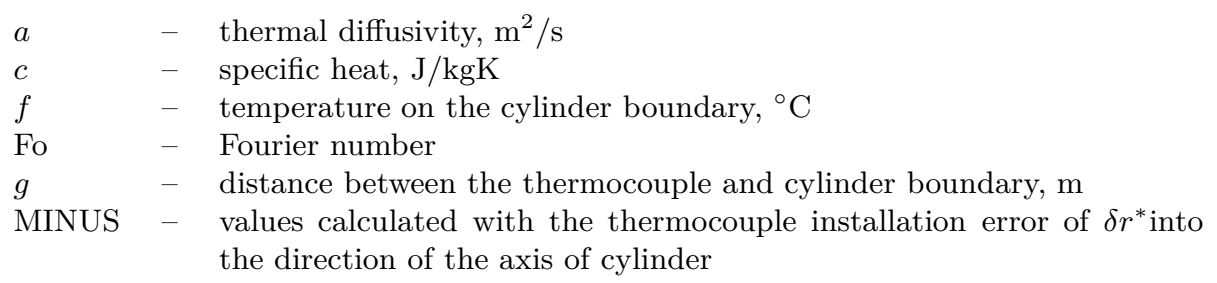

*Corresponding Author. Email magda.joachimiak@put.poznan.pl 


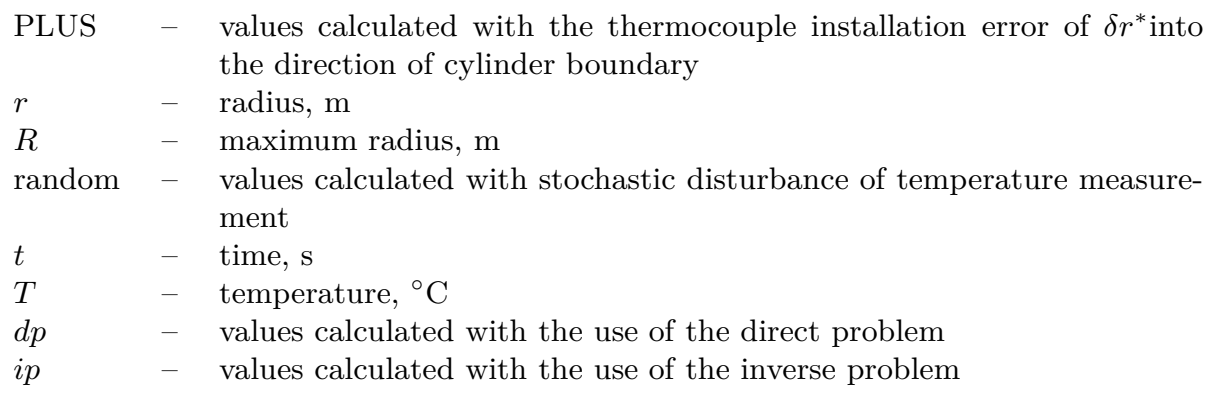

\section{Greek symbols}

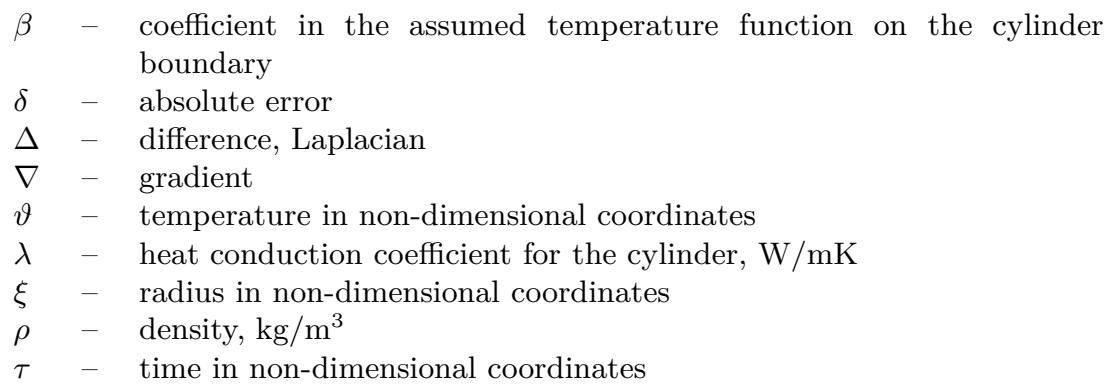

\section{Subscripts}



\section{Superscripts}

* - measuring

\section{Introduction}

Realization of thermal field fulfilling the set criteria is required in processes of heating machine's components. To control body heating, it is important to know the temperature on the boundary of the region. However, it is not always possible to measure the boundary temperature, as, for example, in the burning chamber or on the inner surface of gas-turbine casing. It is extremely difficult when radiation constitutes a great part of heating process (heat treatment processes). In such cases, the boundary temperature can be determined from solution of the inverse problem based on temperature measurement at inner points in the body, arranged close to the boundary 
where the course of temperature is not known $[1,21]$. Some methods of solving one-dimensional inverse problem of thermal field distribution for a cylinder were presented in [3]; and for the cylindrical layer - in [2]. Solution of the inverse problem based on Laplace's transform was discussed in $[2,3,10,12]$. Inverse problem for the heat conduction equation was solved with the use of sequential method and described in papers [2,22]. Analysis of thermal fields during unsteady heat transfer for an irregular geometry was described in [4]. Method of inverse problem finds a wide application in technical issues. In [24], a substitute calculation model for the inverse heat conduction problem was discussed. Boundary condition for a heated beam was sought for using this model and the finite element method. Paper [5] presents the algorithm for solving the inverse heat flow problems, which use the finite element method. The concept of this algorithm consists in solving the Neumann problem, where the heat flux on the inner boundary is sought. Algorithm gave smooth, non-oscillating and stable solution. It was used to analyse heat transfer in the region with holes. Paper [6] presents a method of solving the inverse heat conduction problem, comprising the solution of the Poisson's equation for simpler and linked with each other regions instead of the Laplace's equation for multiply-connected region, such as the gas-turbine blade with cooling channels. In paper [17], by means of solving the heat equation for $2 \mathrm{D}$ model in a steady-state with the use of the inverse problem, thermal conductivity of material as the polynomial depending on temperature was sought for. Paper [14] discusses heat flow in high-temperature industrial furnaces. Solving direct and inverse problems with the use of the conjugate gradient method for the changes of phase of metal solidification were analysed. Trefftz methods as well as the method of fundamental solutions are often used for solving direct and inverse problems for the equation of heat conduction $[13,18,23]$. Paper [8] presents the solution of direct and inverse non-Fourier heat conduction problems with the use of Trefftz functions and Trefftz method. Paper [9] presents the method of solving nonlinear direct and inverse heat conduction problems with the use of Trefftz functions. Paper [16] discusses the solution of the inverse problem for the Poisson equation with the use of fundamental solution method and Tikhonov regularization. Application of the fundamental solutions method for solving the inverse problem of heat source determination in an unsteady heat conduction is presented in [15]. 
Heat treatment and thermo-chemical treatment processes comprise wide ranges of temperature. For nitrification process, heating temperature ranges from an ambient temperature up to the temperature of approx. $550^{\circ} \mathrm{C}$. Considering such range of temperature for steel, any change of heat conduction coefficient, $\lambda$, and specific heat, $c$, is significant. For the analysed range of temperature, the heat conduction coefficient changes from $52 \mathrm{~W} /(\mathrm{mK})$ to $36 \mathrm{~W} /(\mathrm{mK})$, and specific heat changes from $440 \mathrm{~J} /(\mathrm{kgK})$ to $750 \mathrm{~J} /(\mathrm{kgK})$ [11]; it is $30.8 \%$ and $70.5 \%$ of their initial values, respectively Heat conduction equation includes the change of thermal diffusivity $a=\lambda / \rho c$. Hence, the increase of $\lambda$ and decrease of c causes the change of thermal diffusivity coefficient $a$ from $0.000015 \mathrm{~m}^{2} / \mathrm{s}$ to $0.000007 \mathrm{~m}^{2} / \mathrm{s}$, what is $53.3 \%$. Such significant change of thermal diffusivity for temperature ranging from $20{ }^{\circ} \mathrm{C}$ to $550^{\circ} \mathrm{C}$ in heat treatment processes requires application of mathematical model in which $\lambda$ and $\mathrm{c}$ are functions depending on temperature (non-linear problem). Knowledge of temperature distribution during heating enables precise analysis of heat treatment processes and stresses arising during heating. Therefore, non-linear and unsteady heat conduction equation was solved in this paper with the use of the method of inverse problem.

\section{Direct problem}

Solution of direct problem enables determining temperature distribution in a cylinder with the known temperature on its boundary. For the heat conduction equation

$$
\rho(T) c(T) \frac{\partial T}{\partial t}=\operatorname{div}(\lambda(T) \nabla T)
$$

the following initial condition was assumed

$$
T(r, t=0)=T_{0}=0 .
$$

Kirchhoff's substitution [7] was applied

$$
\vartheta=\frac{1}{\lambda_{0}} \int_{T_{0}}^{T} \lambda(u) d u=\frac{1}{\lambda_{0}} \int_{0}^{T} \lambda(u) d u
$$

and

$$
\vartheta+\delta \vartheta=\frac{1}{\lambda_{0}} \int_{0}^{T+\delta T} \lambda(u) d u
$$


After substracting (3) from (4), we obtain

$$
\delta \vartheta=\frac{1}{\lambda_{0}}\left(\int_{0}^{T+\delta T} \lambda(u) d u-\int_{0}^{T} \lambda(u) d u\right)=\frac{1}{\lambda_{0}} \int_{T}^{T+\delta T} \lambda(u) d u .
$$

It was hence obtained [7]

$$
\frac{\partial \vartheta}{\partial t}=a(\vartheta(T)) \Delta \vartheta
$$

Equation (1) in polar coordinates reads

$$
\frac{\partial \vartheta}{\partial t}=a(\vartheta(T))\left(\frac{\partial^{2} \vartheta}{\partial r^{2}}+\frac{1}{r} \frac{\partial \vartheta}{\partial r}\right)
$$

and when the non-dimensional coordinate

$$
\bar{\xi}=\frac{r}{R} \in\langle 0,1\rangle
$$

is introduced the Eq. (2) is transformed to the form

$$
\frac{\partial \vartheta}{\partial t}=a(\vartheta(T)) \frac{1}{R^{2}}\left(\frac{\partial^{2} \vartheta}{\partial \bar{\xi}^{2}}+\frac{1}{\bar{\xi}} \frac{\partial \vartheta}{\partial \bar{\xi}}\right)
$$

It was assumed that density changes a little during the heating process, and heat conduction coefficient and specific heat depend on temperature

$$
\frac{\partial \vartheta}{\partial t}=\frac{\lambda(\vartheta(T))}{\rho_{0} c(\vartheta(T))} \frac{1}{R^{2}}\left(\frac{\partial^{2} \vartheta}{\partial \bar{\xi}^{2}}+\frac{1}{\bar{\xi}} \frac{\partial \vartheta}{\partial \bar{\xi}}\right)
$$

When the non-dimensional time coordinate $\tau=$ Fo $=\frac{a t}{R^{2}}=\frac{\lambda_{0} t}{\rho_{0} c_{0} R^{2}}$ was introduced, the following equation was obtained:

$$
\frac{\partial \vartheta}{\partial \tau}=\frac{\lambda(\vartheta(T))}{\lambda_{0}} \frac{c_{0}}{c(\vartheta(T))}\left(\frac{\partial^{2} \vartheta}{\partial \bar{\xi}^{2}}+\frac{1}{\bar{\xi}} \frac{\partial \vartheta}{\partial \bar{\xi}}\right), \quad \bar{\xi} \in(0,1) .
$$

Since the variable $\bar{\xi} \in\langle 0,1\rangle$, therefore substitution $\xi=2 \bar{\xi}-1$ is done. Then [19]

$$
\bar{\xi}=\frac{\xi+1}{2}, \quad \frac{\partial \vartheta}{\partial \bar{\xi}}=2 \frac{\partial \vartheta}{\partial \xi}, \quad \frac{\partial^{2} \vartheta}{\partial \bar{\xi}^{2}}=4 \frac{\partial^{2} \vartheta}{\partial \xi^{2}}
$$


Hence

$$
\frac{\partial \vartheta}{\partial \tau}=4 \frac{\lambda(\vartheta(T))}{\lambda_{0}} \frac{c_{0}}{c(\vartheta(T))}\left(\frac{\partial^{2} \vartheta}{\partial \xi^{2}}+\frac{1}{\xi+1} \frac{\partial \vartheta}{\partial \xi}\right) .
$$

Approximating the derivative with respect to time by the backward difference quotient, we have obtained the following linear equation:

$$
\frac{\tilde{\vartheta}\left(\tau_{i}\right)-\tilde{\vartheta}\left(\tau_{i-1}\right)}{\Delta \tau} \approx 4 \frac{\lambda\left(\tilde{\vartheta}_{i-1}\right)}{\lambda_{0}} \frac{c_{0}}{c\left(\tilde{\vartheta}_{i-1}\right)}\left(\frac{d^{2} \tilde{\vartheta}}{d \xi^{2}}+\frac{1}{\xi+1} \frac{d \tilde{\vartheta}}{d \xi}\right) .
$$

Solution to Eq. (11) is sought in the form of the linear combination of Chebyshev polynomials

$$
\tilde{\vartheta}=\sum_{k=0}^{N} \alpha_{k} W_{k}(\xi), \quad \xi \in\langle-1,1\rangle
$$

where $W_{k}(\xi)$ denotes the Chebyshev polynomials of the first type [20].

Assuming that $\beta^{2}=\frac{1}{\Delta \tau}, Q=\vartheta\left(\tau_{i-1}\right)$ and taking substitution (15) into account, we obtain the following system of linear equations

$$
\begin{aligned}
\beta^{2} \sum_{k=0}^{N} \alpha_{k} W_{k}(\xi)-\beta^{2} Q & = \\
& 4 \frac{\lambda\left(\tilde{\vartheta}_{i-1}\right)}{\lambda_{0}} \frac{c_{0}}{c\left(\tilde{\vartheta}_{i-1}\right)}\left(\sum_{k=0}^{N} \alpha_{k} W_{k}^{\prime \prime}(\xi)+\frac{1}{\xi+1} \sum_{k=0}^{N} \alpha_{k} W_{k}^{\prime}(\xi)\right) .
\end{aligned}
$$

Let $q(\xi, \tau)=4 \frac{\lambda\left(\vartheta_{i-1}\right)}{\lambda_{0}} \frac{c_{0}}{c\left(\vartheta_{i-1}\right)}$, thus we have

$$
q(\xi, \tau) \sum_{k=0}^{N} \alpha_{k} W_{k}^{\prime \prime}(\xi)+\frac{1}{\xi+1} q(\xi, \tau) \sum_{k=0}^{N} \alpha_{k} W_{k}^{\prime}(\xi)-\beta^{2} \sum_{k=0}^{N} \alpha_{k} W_{k}(\xi)=-\beta^{2} Q .
$$

Therefore,

$$
\sum_{k=0}^{N} \alpha_{k} q(\xi, \tau) W_{k}^{\prime \prime}(\xi)+\sum_{k=0}^{N} \alpha_{k} \frac{1}{\xi+1} q(\xi, \tau) W_{k}^{\prime}(\xi)-\sum_{k=0}^{N} \alpha_{k} \beta^{2} W_{k}(\xi)=-\beta^{2} Q .
$$

Hence, assuming $p(\xi)=\frac{1}{\xi+1}$ and $r(\xi, \tau)=-\beta^{2} Q$, we have

$$
\sum_{k=0}^{N} \alpha_{k}\left(q(\xi, \tau) W_{k}^{\prime \prime}(\xi)+p(\xi) q(\xi, \tau) W_{k}^{\prime}(\xi)-\beta^{2} W_{k}(\xi)\right)=r(\xi, \tau) .
$$


To determine coefficients $\alpha_{k}$, the collocation method is used, then for the radius $\xi_{i}$ (for a period of time), we obtain the equation

$$
\sum_{k=0}^{N} \alpha_{k}\left(q\left(\xi_{i}\right) W_{k}^{\prime \prime}\left(\xi_{i}\right)+p\left(\xi_{i}\right) q\left(\xi_{i}\right) W_{k}^{\prime}\left(\xi_{i}\right)-\beta^{2} W_{k}\left(\xi_{i}\right)\right)=r\left(\xi_{i}\right)
$$

and demand that Eq. (20) is satisfied at all inner points $\xi_{i}(i=1,2, \ldots$, $N-1$ ). Hence we have $\mathrm{N}-1$ of equations, and the number of unknowns is $\mathrm{N}$ $+1, \alpha_{0}, \alpha_{1}, \ldots, \alpha_{N}$; to close the system of equations, boundary conditions are joined

$$
\begin{gathered}
\left.\frac{\partial \tilde{\vartheta}}{\partial \xi}\right|_{\xi=-1}=0, \\
\left.\tilde{\vartheta}\right|_{\xi=1}=f(\tau),
\end{gathered}
$$

which, based on (15), has the form

$$
\begin{aligned}
& \left.\frac{\partial \tilde{\vartheta}}{\partial \xi}\right|_{\xi=\xi_{0}=-1}=\sum_{k=0}^{N} \alpha_{k} W_{k}^{\prime}\left(\xi_{0}=-1\right)=0, \\
& \left.\tilde{\vartheta}\right|_{\xi=\xi_{N}=1}=\sum_{k=0}^{N} \alpha_{k} W_{k}\left(\xi_{N}=1\right)=f(\tau) .
\end{aligned}
$$

Equation (20) together with boundary conditions (23) and (24) creates the following matrix equation

$$
A_{(N+1) \times(N+1)}\left\{\begin{array}{c}
\alpha_{0} \\
\alpha_{1} \\
\vdots \\
\alpha_{N-1} \\
\alpha_{N}
\end{array}\right\}=\left\{\begin{array}{c}
0 \\
r\left(\xi_{1}\right) \\
\vdots \\
r\left(\xi_{N-1}\right) \\
f
\end{array}\right\} .
$$

Elements of matrix $\mathbf{A}$ are expressed by the formulae

$$
\begin{gathered}
a_{0 j}=W_{j}^{\prime}\left(\xi_{0}=-1\right) \\
a_{i j}=q\left(\xi_{i}\right) W_{j}^{\prime \prime}\left(\xi_{i}\right)+p\left(\xi_{i}\right) q\left(\xi_{i}\right) W_{j}^{\prime}\left(\xi_{i}\right)-\beta^{2} W_{j}\left(\xi_{i}\right) \\
a_{N j}=W_{j}\left(\xi_{N}=1\right)
\end{gathered}
$$


for $i=1,2, \ldots, N-1, j=0,1,2, \ldots, N$. Multiplying the left side of Eq. (25) by the matrix $A^{-1}$, we obtain

$$
\left\{\begin{array}{c}
\alpha_{0} \\
\alpha_{1} \\
\vdots \\
\alpha_{N-1} \\
\alpha_{N}
\end{array}\right\}=A^{-1}\left\{\begin{array}{c}
0 \\
r\left(\xi_{1}\right) \\
\vdots \\
r\left(\xi_{N-1}\right) \\
f
\end{array}\right\},
$$

what can be noted as

$$
\left\{\begin{array}{c}
\alpha_{0} \\
\alpha_{1} \\
\vdots \\
\alpha_{N-1} \\
\alpha_{N}
\end{array}\right\}=\left[\begin{array}{ccccc}
\tilde{a}_{00} & \tilde{a}_{01} & \ldots & \ldots & \tilde{a}_{0 N} \\
\tilde{a}_{10} & \tilde{a}_{11} & \ldots & \ldots & \tilde{a}_{1 N} \\
\vdots & \vdots & \ddots & & \vdots \\
\vdots & \vdots & & \ddots & \vdots \\
\tilde{a}_{N 0} & \tilde{a}_{N 1} & \ldots & \ldots & \tilde{a}_{N N}
\end{array}\right]\left\{\begin{array}{c}
0 \\
r\left(\xi_{1}\right) \\
\vdots \\
r\left(\xi_{N-1}\right) \\
f
\end{array}\right\}
$$

where $\tilde{a}_{i j}$ are elements of the matrix $\mathbf{A}^{-1}$.

\section{Inverse problem}

The purpose of solving the inverse problem is to determine unknown temperature on the boundary of the cylinder $f$ (in subsequent moments of time) based on temperature measurements inside the cylinder and on the form of the solution to the direct problem. On the basis of the Eq. (30) we have that

$$
\left\{\begin{array}{c}
\alpha_{0} \\
\alpha_{1} \\
\vdots \\
\alpha_{N-1} \\
\alpha_{N}
\end{array}\right\}=\left\{\begin{array}{c}
\sum_{i=1}^{N-1} \tilde{a}_{0 i} r\left(\xi_{i}\right)+f \tilde{a}_{0 N} \\
\sum_{i=1}^{N-1} \tilde{a}_{1 i} r\left(\xi_{i}\right)+f \tilde{a}_{1 N} \\
\vdots \\
\vdots \\
\sum_{i=1}^{N-1} \tilde{a}_{N i} r\left(\xi_{i}\right)+f \tilde{a}_{N N}
\end{array}\right\}
$$

Therefore,

$$
\alpha_{j}=\sum_{i=1}^{N-1} \tilde{a}_{j i} r\left(\xi_{i}\right)+f \tilde{a}_{j N} \quad \text { for } \quad j=0,1,2, \ldots, N .
$$

Coefficients $\alpha_{j}$ were introduced into the solution (15). Hence,

$$
\tilde{\vartheta}(\xi)=\sum_{k=0}^{N}\left(\sum_{i=1}^{N-1} \tilde{a}_{k i} r\left(\xi_{i}\right)+f \tilde{a}_{k N}\right) W_{k}(\xi) .
$$




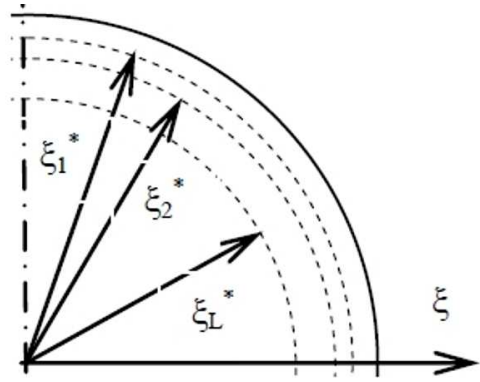

Figure 1: Measuring points.

For measuring points $\xi_{l}^{*}$, where $l=1,2, \ldots, L$ (Fig. 1), we have that

$$
\tilde{\vartheta}\left(\xi_{l}^{*}\right)=\sum_{k=0}^{N}\left(\sum_{i=1}^{N-1} \tilde{a}_{k i} r\left(\xi_{i}\right)+f \tilde{a}_{k N}\right) W_{k}\left(\xi_{l}^{*}\right) .
$$

To determine unknown parameters $\alpha_{0}, \alpha_{1}, \ldots, \alpha_{N}$, the functional should be minimized

$$
I(f)=\sum_{l=1}^{L}\left(\tilde{\vartheta}\left(\xi_{l}^{*}, f\right)-\tilde{\vartheta}_{m}\left(\xi_{l}^{*}\right)\right)^{2} .
$$

Having substituted formula (33), for the value calculated at measuring points $\xi_{l}^{*}$, where $l=1,2, \ldots, L$, we have

$$
I(f)=\sum_{l=1}^{L}\left[\sum_{k=0}^{N}\left(\sum_{i=1}^{N-1} \tilde{a}_{k i} r\left(\xi_{i}\right)+f \tilde{a}_{k N}\right) W_{k}\left(\xi_{l}^{*}\right)-\tilde{\vartheta}_{m}\left(\xi_{l}^{*}\right)\right]^{2} .
$$

Annihilation of the first derivative is the necessary condition of the functional (4) minimum

$$
\frac{d I}{d f}=0
$$

Including Eqs. (4) and (37), we obtained

$$
\sum_{l=1}^{L}\left\{\left[\sum_{k=0}^{N}\left(\sum_{i=1}^{N-1} \tilde{a}_{k i} r\left(\xi_{i}\right)+f \tilde{a}_{k N}\right) W_{k}\left(\xi_{l}^{*}\right)-\tilde{\vartheta}_{m}\left(\xi_{l}^{*}\right)\right] \sum_{k=0}^{N} W_{k}\left(\xi_{l}^{*}\right) \tilde{a}_{k N}\right\}=0 .
$$


Hence,

$$
\begin{gathered}
\sum_{l=1}^{L}\left\{\left[\sum_{k=0}^{N} W_{k}\left(\xi_{l}^{*}\right)\left(\sum_{i=1}^{N-1} \tilde{a}_{k i} r\left(\xi_{i}\right)\right)-\tilde{\vartheta}_{m}\left(\xi_{l}^{*}\right)\right] \sum_{k=0}^{N} W_{k}\left(\xi_{l}^{*}\right) \tilde{a}_{k N}+\right. \\
\left.f\left(\sum_{k=0}^{N} W_{k}\left(\xi_{l}^{*}\right) \tilde{a}_{k N}\right)^{2}\right\}=0 .
\end{gathered}
$$

Let

$$
\begin{gathered}
A_{l}=\left[\sum_{k=0}^{N} W_{k}\left(\xi_{l}^{*}\right)\left(\sum_{i=1}^{N-1} \tilde{a}_{k i} r\left(\xi_{i}\right)\right)-\tilde{\vartheta}_{m}\left(\xi_{l}^{*}\right)\right] \sum_{k=0}^{N} W_{k}\left(\xi_{l}^{*}\right) \tilde{a}_{k N}, \\
B_{l}=\left(\sum_{k=0}^{N} W_{k}\left(\xi_{l}^{*}\right) \tilde{a}_{k N}\right)^{2}
\end{gathered}
$$

then the Eq. (39) can be noted as

$$
\sum_{l=1}^{L}\left\{A_{l}+f B_{l}\right\}=0
$$

Therefore,

$$
f=\frac{-\sum_{l=1}^{L} A_{l}}{\sum_{l=1}^{L} B_{l}} .
$$

In mathematical model, the heat conduction coefficient depends on temperature, and can be noted as the linear combination of Chebyshev polynomials

$$
\lambda(T)=\sum_{i=0}^{n} a_{i} W_{i}(\tilde{T}),
$$

where temperature $\tilde{T}=\frac{2 T-T_{\max }-T_{0}}{T_{\max }-T_{0}}$, then $\tilde{T} \in\langle-1,1\rangle$. Applying the Kirchhoff's substitution (3), we obtain

$$
\vartheta=\frac{1}{\lambda_{0}} \int_{T_{0}}^{T} \lambda(u) d u=\frac{T_{\max }-T_{0}}{2 \lambda_{0}} \int_{T_{0}}^{T} \sum_{i=0}^{n} a_{i} W_{i}(\tilde{T}) d \tilde{T} .
$$

Since $\vartheta=g(T)$, hence $T=g^{-1}(\vartheta)$. For the value $\vartheta(T)$, the interval $\left\langle T_{i}, T_{i+1}\right\rangle$ (Fig. 2), for which the following inequality is satisfied, is sought

$$
\vartheta\left(T_{i}\right) \leq \vartheta(T) \leq \vartheta\left(T_{i+1}\right) .
$$




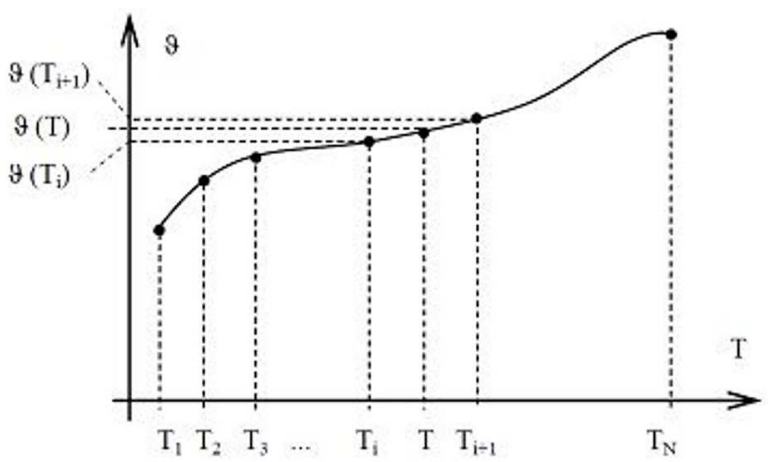

Figure 2: Determination of temperature $\vartheta(T)$ intermediate value in $\left(T_{i}, T_{i+1}\right)$ interval in the function of $k$ parameter, Eq. (48).

Then, on the basis of linear extrapolation

$$
\begin{gathered}
\vartheta(T)=k \vartheta\left(T_{i}\right)+(1-k) \vartheta\left(T_{i+1}\right), \\
T=k T_{i}+(1-k) T_{i+1} .
\end{gathered}
$$

Hence,

$$
k=\frac{\vartheta(T)-\vartheta\left(T_{i+1}\right)}{\vartheta\left(T_{i}\right)-\vartheta\left(T_{i+1}\right)} .
$$

Specific heat was also presented as the linear combination of Chebyshev polynomials

$$
c(T)=\sum_{i=0}^{n} b_{i} W_{i}(\tilde{T}),
$$

where temperature $\tilde{T} \in\langle-1,1\rangle$. Coefficients $\mathrm{a}_{i}$ and $\mathrm{b}_{i}$ were determined with the use of the least squares approximation method [14]. They are presented in Tab. 1.

\section{Sensitivity of the solution to the inverse problem to errors in measurements}

To determine the distribution of temperature on the cylinder boundary, it is necessary to measure temperature inside this cylinder as close to the boundary as possible. Sensitivity of the solution to the inverse problem comprises the impact of the thermocouple installation error $\pm \delta \xi_{l}^{*}$ as well 
Table 1: Coefficients of polynomials approximating functions $\lambda(\mathrm{T})$ and $\mathrm{c}(\mathrm{T})$.

\begin{tabular}{|c|c|c|}
\hline $\mathrm{i}$ & \multicolumn{1}{|c|}{$\mathrm{a}_{i}$} & $\mathrm{~b}_{i}$ \\
\hline 0 & 41.519 & 660.920 \\
\hline 1 & -11.350 & 247.360 \\
\hline 2 & -0.90257 & 51.725 \\
\hline 3 & 0.010553 & 18.980 \\
\hline
\end{tabular}

as the error in temperature measurement $\delta \vartheta_{l}=h\left(\delta T_{i}\right)(5)$ on the sought distribution of temperature on the cylinder boundary. Non-dimensional temperature on the boundary is

$$
f_{ \pm \delta \xi_{l}^{*}, \delta \vartheta}=\frac{-\sum_{l=1}^{L} A_{l, \pm \delta \xi_{l}^{*}, \delta \vartheta_{l}}}{\sum_{l=1}^{L} B_{l, \pm \delta \xi_{l}^{*}, \delta \vartheta_{l}}},
$$

where

$$
\begin{aligned}
& A_{l, \pm \delta \xi_{l}^{*}, \delta \vartheta_{l}}= {\left[\sum_{k=0}^{N} W_{k}\left(\xi_{l}^{*}\right)\left(\sum_{i=1}^{N-1} \tilde{a}_{k i} r\left(\xi_{i}\right)\right)-\right.} \\
&\left.\left(\tilde{\vartheta}_{m}\left(\xi_{l}^{*} \pm \delta \xi_{l}^{*}\right)+\delta \vartheta_{l}\right)\right] \sum_{k=0}^{N} W_{k}\left(\xi_{l}^{*}\right) \tilde{a}_{k N} \\
& B_{l, \pm \delta \xi_{l}^{*}, \delta \vartheta_{l}}=B_{l}=\left(\sum_{k=0}^{N} W_{k}\left(\xi_{l}^{*}\right) \tilde{a}_{k N}\right)^{2} .
\end{aligned}
$$

\section{$5 \quad$ Numerical example}

To test the program, it was assumed that the distribution of temperature on the cylinder boundary can be described by the function of the form $f(\tau)=T_{\max }\left(1-e^{-\beta \tau}\right)$, where $\beta=1.1$. Calculations were performed for the cylinder of $100 \mathrm{~mm}$ diameter. The following values were assumed for calculations: $\lambda_{0}=52.531 \frac{\mathrm{W}}{\mathrm{mK}}, c_{0}=429.331 \frac{\mathrm{J}}{\mathrm{kgK}}$, and $\Delta \tau=0.187$. Direct problem was solved and distributions of temperature on radii $r_{1}=48 \mathrm{~mm}$, 




a)

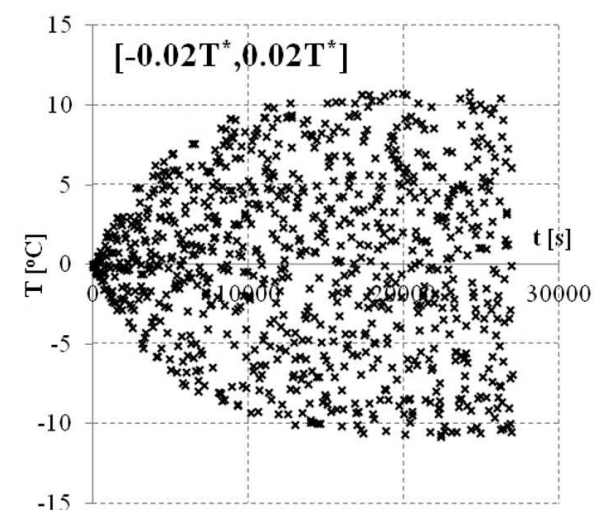

b)

Figure 3: Course of the random error in temperature measurement of values from the interval $\left[-0.01 T^{*}, 0.01 T^{*}\right](\mathrm{a})$, and $\left[-0.02 T^{*}, 0.02 T^{*}\right](\mathrm{b})$ for the temperature measurement at the distance of $2 \mathrm{~mm}$ from the boundary of the cylinder with the installation error $\delta r^{*}=-0.5 \mathrm{~mm}$.
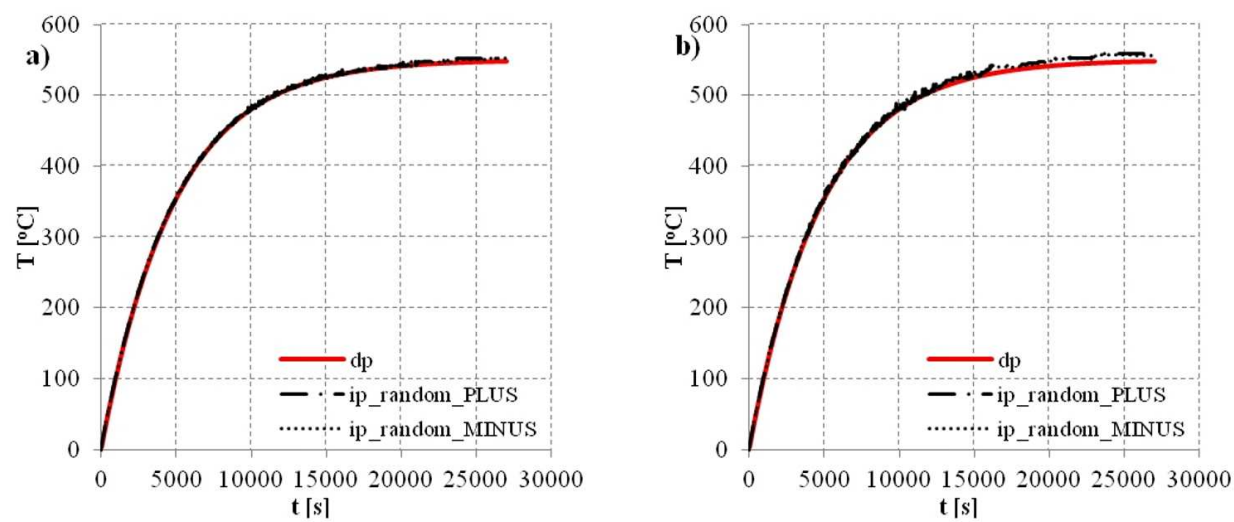

Figure 4: Distribution of temperature on the boundary of the cylinder assumed in the direct problem $(d p)$ as well as calculated with the use of the inverse problem (ip) including error in thermocouple installation (PLUS, MINUS) and random error in temperature measurement (random): a) $0.01 \mathrm{~T}^{*}$, b) $0.02 \mathrm{~T}^{*}$.

$r_{2}=46 \mathrm{~mm}$ and $r_{3}=44 \mathrm{~mm}\left(g_{1}=2 \mathrm{~mm}, g_{2}=4 \mathrm{~mm}\right.$ and $\left.g_{3}=6 \mathrm{~mm}\right)$ were determined. Obtained temperature values were input data for the solution of inverse problem and corresponded to the temperature measured by thermocouples. Analysis of solution sensitivity to errors in thermocouple installation was conducted. It was assumed that each of thermocouples may 
be shifted into the direction of the cylinder boundary by $\delta r^{*}=0.5 \mathrm{~mm}$, what was noted as PLUS in Fig. 3. Shifting thermocouple closer to the cylinder axis corresponds to the error in installation $\delta r^{*}=-0.5 \mathrm{~mm}$ and is noted as MINUS (Fig. 4). In calculations, random error in temperature measurement $\delta T^{*}$ was included, what is denoted as random in Fig. 3. This error was a random number from the interval $\left[-0.01 T^{*}, 0.01 T^{*}\right]$ or $\left[-0.02 T^{*}, 0.02 T^{*}\right]$. Figure 3 presents illustrative course of the random error in temperature measurement for the thermocouple located at the distance of $2 \mathrm{~mm}$ from the boundary with the installation error $\delta r^{*}=-0.5 \mathrm{~mm}$ and with the maximal errors in temperature measurement $\delta T^{*}=0.01 T^{*}$ and $\delta T^{*}=0.02 T^{*}$.

Distributions of temperature on the boundary of the cylinder, assumed in the direct problem and calculated with the use of inverse problem, including the sensitivity of the solution were presented in Fig. 4. For the random error in temperature measurement $\delta T^{*}=0.01 \mathrm{~T}^{*}$ and for thermocouples shifted into the direction of the cylinder axis by $\left|\delta r^{*}\right|=0.5 \mathrm{~mm}$, the error of temperature distribution on the cylinder boundary was slightly above $6{ }^{\circ} \mathrm{C}$ (Fig. 5a, b). Larger differences between the assumed temperature and the calculated one with the use of the inverse problem were obtained when the random disturbance in temperature measurement was up to $0.02 T^{*}$. Those values are up to $13{ }^{\circ} \mathrm{C}$ (Fig. 5c, d).

Temperature distributions in the cylinder, assumed in the direct problem and calculated with the use of the inverse problem method for the time of 900 s, 2100 s, 3000 s, 4200 s, 5100 s, and 8100 s are presented in Fig. 6. Calculations were made for thermocouples located at the distance of 2,4 and $6 \mathrm{~mm}$ from the boundary. Installation error was $\delta r^{*}=-0.5 \mathrm{~mm}$. Random error in temperature measurement reached maximally $2 \%$ of the measured value, what correspond to values from the interval $\left[-0.02 T^{*}, 0.02 T^{*}\right]$.

Figure 7 presents the assumed distribution of temperature on the boundary of the cylinder as well as the distributions of temperature calculated with the use of the inverse problem for thermocouples located at the distance of 2,4 and $6 ; 4,6$, and 8 as well as 6,8 , and $10 \mathrm{~mm}$ from the boundary. For random error in temperature measurement up to $0.02 T^{*}$, when the distance of thermocouple location from the boundary increases, the maximal error in calculations on the cylinder boundary increases too.

Numerical tests, presented above, assumed greater measurement disturbances than those occurring real production conditions. Figure 8 presents distributions of temperature obtained as a result of solution of the in- 

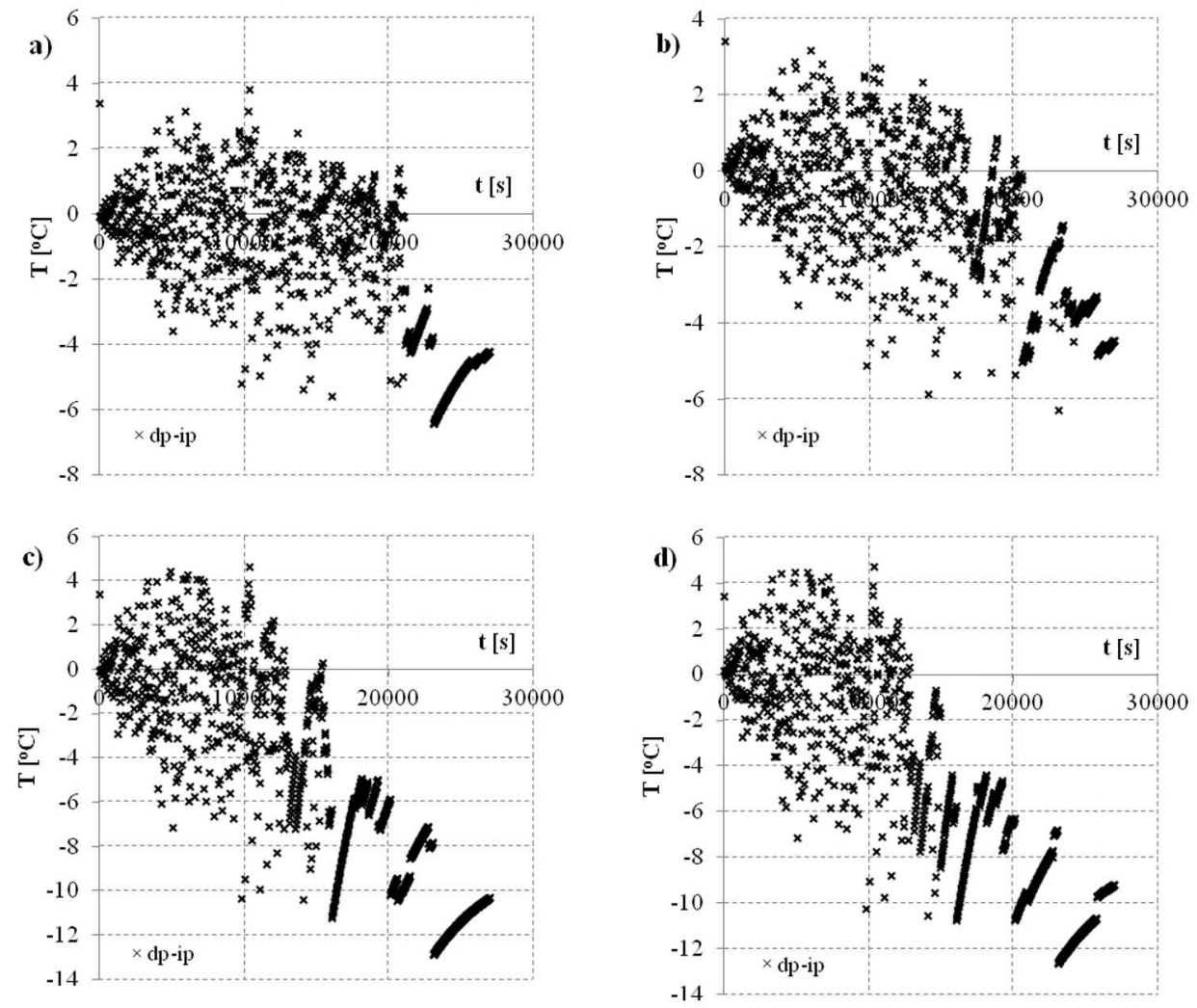

Figure 5: Difference in temperatures on the boundary of the cylinder assumed in the direct problem $(d p)$ and calculated with the use of the inverse problem $(i p)$, including the thermocouple installation errors $\delta r^{*}=0.5 \mathrm{~mm}(\mathrm{a}, \mathrm{c})$ and $\delta r^{*}=$ $-0.5 \mathrm{~mm}(\mathrm{~b}, \mathrm{~d})$ as well as the random error in temperature measurement up to $0.01 T^{*}(\mathrm{a}, \mathrm{b})$ and $0.02 T^{*}(\mathrm{c}, \mathrm{d})$.

verse problem, with the installation error $\delta r^{*}=0.5 \mathrm{~mm}$ (PLUS) and $\delta r^{*}=-0.5 \mathrm{~mm}$ (MINUS). Stable during the whole heating process error in temperature measurement of $3^{\circ} \mathrm{C}$ (PLUS) or $-3^{\circ} \mathrm{C}$ (MINUS) was assumed. These parameters correspond to heating conditions obtainable during the experiment. Errors in temperature distribution, obtained in this experiment, slightly exceeded $3{ }^{\circ} \mathrm{C}$. 



Figure 6: Temperature distribution along the radius of the cylinder for the time of a) $t=900 \mathrm{~s}$, b) $t=2100 \mathrm{~s}$, c) $t=3000 \mathrm{~s}$, d) $t=4200 \mathrm{~s}$, e) $t=5100 \mathrm{~s}$, f) $t=8100 \mathrm{~s}$ with the maximal disturbance of $0.02 \mathrm{~T}^{*}$ and thermocouples located at the distance of 2,4 , and $6 \mathrm{~mm}$ from the boundary with the instalaltion error $\delta r^{*}=-0.5 \mathrm{~mm}$. 

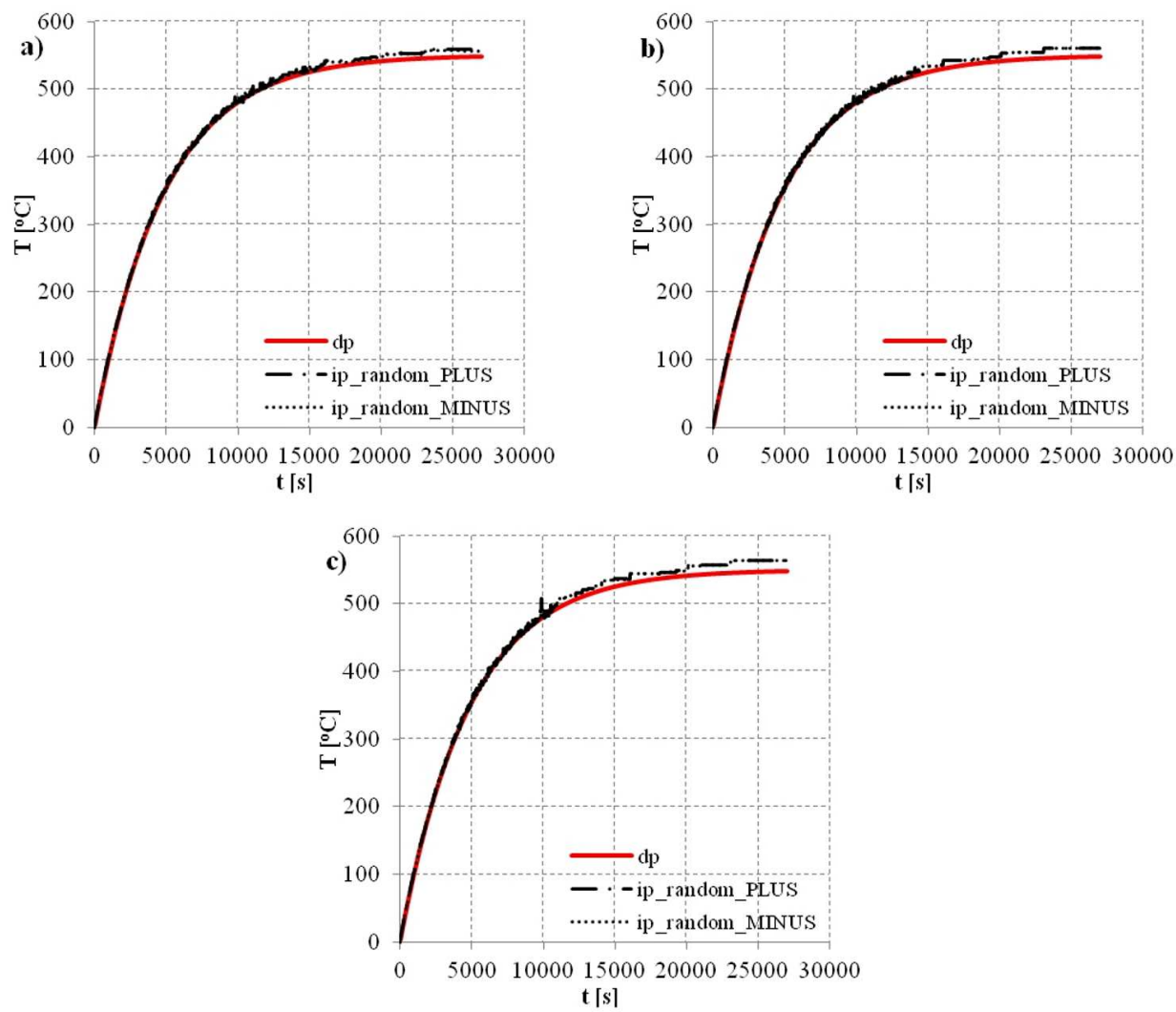

Figure 7: Temperature distribution on the boundary of the cylinder assumed in the direct problem $(d p)$ and calculated with the use the inverse problem $(i p)$ for installation errors $\delta r^{*}=0.5 \mathrm{~mm}$ (PLUS) and $\delta r^{*}=-0.5 \mathrm{~mm}$ (MINUS) with the temperature measurement error up to $0.02 T^{*}$ and thermocouples located at the distance of: a) 2, 4,6 $\mathrm{mm}$; b) 4, 6, $8 \mathrm{~mm}$; c) $6,8,10 \mathrm{~mm}$ from the boundary of the cylinder.

\section{Conclusion}

In heat treatment processes, such as nitrification and carburising, heat conduction coefficient and specific heat change significantly during heating. In the computational model, described in this paper, the change in heat conduction coefficient and specific heat with respect of temperature were included. It enables temperature distribution on the boundary of the cylinder to be determined more precisely by solving the inverse problem and then by solving the direct problem inside the cylinder. It is a basis of pre- 

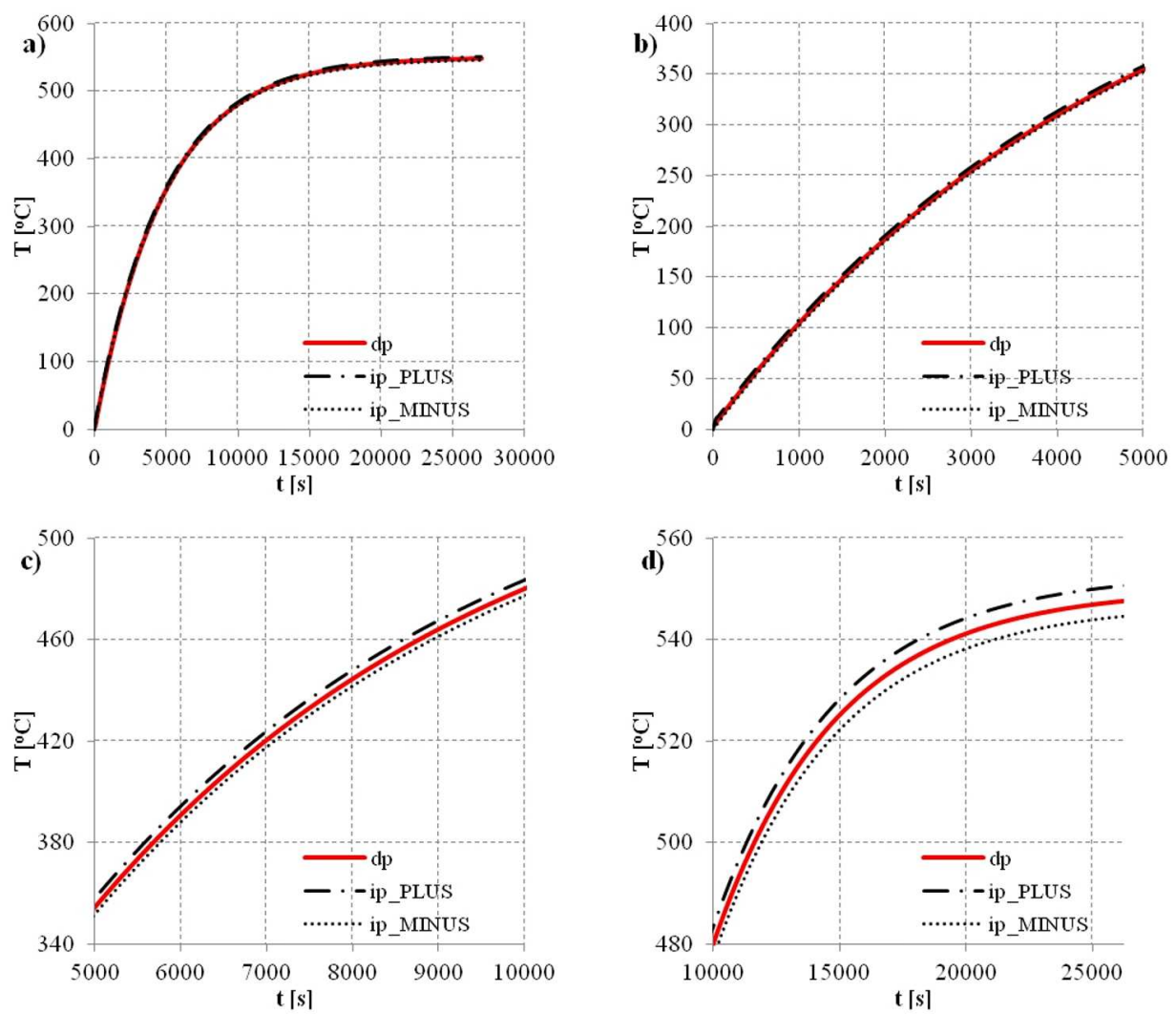

Figure 8: Distribution of temperatue on the boundary of the cylinder assumed in the direct problem $(d p)$ and calculated with use of the inverse problem (ip) for installation errors $\delta r^{*}=0.5 \mathrm{~mm}$ (PLUS) and $\delta r^{*}=-0.5 \mathrm{~mm}$ (MINUS) with the error in temperature measurement $\delta T^{*}=3{ }^{\circ} \mathrm{C}$ (PLUS) and $\delta T^{*}=-3{ }^{\circ} \mathrm{C}$ (MINUS) and thermocouples located at the distance of 2,4 and $6 \mathrm{~mm}$ from the boundary of the cylinder.

cise analysis of structure of the layer, being the subject of heat treatment, and of stresses arising in the component being heat treated. Obtained results indicate low sensitivity of the solution to the inverse problem to the thermocouple installation and temperature measurement errors. 


\section{References}

[1] CiąKowski M.: Selected methods and algorithms for solving inverse problems of heat conduction equation. Wydawnictwo Politechniki Poznańskiej, Poznań 1996 (in Polish).

[2] Ciatkowski M., Grysa K.: A sequential and global method of solving an inverse problem of heat conduction equation. J. Theor. App. Mech-Pol. 48(2010), 1, 111-134.

[3] CiaŁkowski M.J., Grysa K.W.: On a certain inverse problem of temperature and thermal stress fields. Acta Mechanica 36(1980),3-4169-185.

[4] Duda P., TAler J.: Numerical method for the solution of non - linear two - dimensional inverse heat conduction problem using unstructured meshes. Int. J. Numer. Meth. Engng. 48(2000), 7, 881-899, 2000.

[5] Frąckowiak A., Botkin N.D., Ciąkowski M., Hoffmann K.H.: A fitting algorithm for solving inverse problems of heat conduction. Int. J. Heat Mass Trans. 53(2010), 9-10, 2123-2127.

[6] Frackowiak A., Wolfersdorf J.V., Cialkowski M.: Solution of the inverse heat conduction problem described by the Poisson equation for a cooled gas-turbine blade. Int. J. Heat . Mass Trans. 54(2011), 5-6, 1236-1243.

[7] Gdula S.: Heat Conduction. PWN, Warszawa 1984 (in Polish).

[8] Grysa K., MaciąG A., Adamczyk-Krasa J.: Trefftz functions applied to direct and inverse non-fourier heat conduction problems. J. Heat Trans. 136(2014), 9, 1-9.

[9] Grysa K., MaciąG A., Pawinska A.: Solving nonlinear direct and inverse problems of stationary heat transfer by using Trefftz functions. Int. J. Heat Mass Trans. 55(2012), 23-24, 7336-7340.

[10] Han-Taw Chen, Xin-Yi Wu: Investigation of heat transfer coefficient in twodimensional transient inverse heat conduction problems using the hybrid inverse scheme. Int. J. Numer. Meth. Engng. 73(2008), 1, 107-122.

[11] Incropera F.P., De Witt D.P.: Fundamentals of Heat and Mass Transfer. John Wiley \& Sons, New York 1996.

[12] Joachimiak M., CiaŁkowski M.: Optimal choice of integral parameter in a process of solving the inverse problem for heat equation. Arch. Thermodyn. 35(2014), 3, $265-280$.

[13] MaciąG A.: Trefftz functions for selected direct and inverse problems of mechanics. Polit. Świętokrzyska Publishers, Kielce 2009 (in Polish).

[14] Marois M.A., M. DÉsilets M., Lacroix M.: What is the most suitable fixed grid solidification method for handling time-varying inverse Stefan problems in high temperature industrial furnaces? Int. J. Heat Mass Trans. 55(2012), 21-22, 54715478.

[15] Mierzwiczak M., KoŁodziej J.A.: Application of the method of fundamental solutions with the Laplace transformation for the inverse transient heat source problem. J. Theor. App. Mech-Pol. 50(2012), 4, 1011-1023.

[16] Mierzwiczak M., Koøodziej J.A.: The determination of heat sources in two dimensional inverse steady heat problems by means of the method of fundamental solutions. Inverse Prob. Sci. En. 19(2011), 6, 777-792. 
[17] Mierzwiczak M., KoŁodziej J.A.: The determination temperature-dependent thermal conductivity as inverse steady heat conduction problem. Int. J. Heat Mass Trans. 54(2011), 4, 790-796.

[18] Mierzwiczak M., Kolodziej J., CiaŁkowski M., Frąckowiak A.: Implementation of the method of fundamental solutions for heat conduction equation. Poznan University of technology Publishers, Poznań 2011 (in Polish).

[19] Niedoba J., Niedoba W.: Ordinary and partial differential equations. Mathematical problems. (B. Choczewski, Ed.), Uczelniane Wydawnictwo NaukowoDydaktyczne, Kraków 2001 (in Polish).

[20] PAszkowski S.: Numerical implementation of polynominals and Chebyshev. PWN, Warszawa 1975 (in Polish).

[21] TAler J., Duda P.: Solving direct and inverse problems of heat conduction. WNT, Warszawa 2003 (in Polish).

[22] Taler J., Zima W.: Solution of inverse heat conduction problems using control volume approach. Int. J. Heat Mass Trans. 42(1999), 1123-1140.

[23] Trefftz E.: Ein Gegenstük zum Ritz'schenVerfahren. Proc. 2nd Int. Cong. of Applied Mechanics, Zürich 1926, 131-137.

[24] Vakili S., Gadala M.S.: Low cost surrogate model based evolutionary optimization solvers for inverse heat conduction problem. Int. J. Heat Mass Trans. 56(2013), 263273. 